\title{
Pneumatic hiatus
}

\section{Mathew Wedel}

\section{Source}

Mathew John Wedel. (2013). Postcranial pneumaticity in dinosaurs and the origin of the avian lung.

A pneumatic hiatus is an apneumatic portion of the vertebral column that is bordered by pneumatic vertebrae both anteriorly and posteriorly. 